Article

\title{
In Vitro Stimulation of Multidrug Resistance-Associated Protein 2 Function Is Not Reproduced In Vivo in Rats
}

\author{
Ravindranath Reddy Gilibili ${ }^{1,+}$, Vishwanath Kurawattimath ${ }^{1,+}$, Bokka Venkata Murali ${ }^{1}$, \\ Yurong Lai ${ }^{2}$, T. Thanga Mariappan ${ }^{1}$, Hong Shen ${ }^{3}$ and Sagnik Chatterjee ${ }^{1, *}$ \\ 1 Pharmaceutical Candidate Optimization, Biocon Bristol-Myers Squibb R\&D Center (BBRC), \\ Syngene International Ltd., Bangalore 560100, India; ravindra.reddy@syngeneintl.com (R.R.G.); \\ Vishwanath.Kurawattimath@syngeneintl.com (V.K.); Venkata.murali@syngeneintl.com (B.V.M.); \\ Thanga.Mariappan@syngeneintl.com (T.T.M.) \\ 2 Department of drug metabolism, Gilead Sciences Inc., Foster City, CA 94404, USA; Yurong.Lai@gilead.com \\ 3 Pharmaceutical Candidate Optimization, Bristol-Myers Squibb Company, 3551 Lawrenceville Road, \\ Princeton, NJ 08540, USA; hong.shen1@bms.com \\ * Correspondence: sagnik.chatterjee@syngeneintl.com; Tel.: +91-80-6633-4084 \\ + These authors contributed equally to this work.
}

Received: 29 June 2018; Accepted: 31 July 2018; Published: 8 August 2018

\begin{abstract}
Previously we reported that coproporphyrin-I (CP-I) is an optimal probe substrate for multidrug resistance-associated protein 2 (MRP2), and stimulation of MRP2-mediated transport is probe substrate-dependent. In the present investigation, we assessed if the in vitro stimulation is physiologically relevant. Similar to human MRP2 transport, CP-I was transported by rat Mrp2 in a typical Michaelis-Menten kinetics with apparent $K_{m}$ and $V_{\max }$ values of $15 \pm 6 \mu \mathrm{M}$ and $161 \pm$ $20 \mathrm{pmol} / \mathrm{min} / \mathrm{mg}$ protein, respectively. In vivo Mrp2 functions were monitored by biliary and renal secretion of CP-I and its isomer CP-III, in bile-duct cannulated rats before and after treatment with mitoxantrone, progesterone, and verapamil. These compounds stimulated Mrp2-mediated CP-I transport in vitro. No significant increase in biliary or renal clearances, as well as in the cumulative amount of CP-I or CP-III eliminated in bile, were detected following treatment with the in vitro stimulators, indicating an in vitro to in vivo disconnect. In presence of $10 \mu \mathrm{M}$ bilirubin, the in vitro stimulation was suppressed. We concluded that the in vitro stimulation of CP-I transport mediated by Mrp2 is not translatable in vivo, and proposed that the presence of endogenous compounds such as bilirubin in the liver may contribute to the in vitro to in vivo disconnect.
\end{abstract}

Keywords: $\mathrm{ABCC} 2$; MRP2; in vitro stimulation; drug transporter; coproporphyrin

\section{Introduction}

It is becoming increasingly evident that multidrug resistance-associated protein 2 (MRP2/Mrp2, denoted by gene $\mathrm{ABCC} 2 / \mathrm{Abcc} 2$ ) plays an important role in disposition and elimination to regulate pharmacokinetics and pharmacodynamic of xenobiotics [1,2]. MRP2 is located in the canalicular membrane of hepatocytes but also in the apical (luminal) membrane of enterocytes and renal proximal tubule cells [3]. In hepatocytes, MRP2 facilitates the efflux of glucuronide and glutathione conjugates of endobiotics, such as bilirubin conjugates and dianionic bile acids [4], as well as few anionic xenobiotics, such as methotrexate [5]. The inhibition of MRP2 activity is therefore important for drug hepatic disposition and elimination, and can cause potential drug-drug interactions (DDI) and hepatotoxicity [6]. For example, a two-fold increase in methotrexate plasma levels due to MRP2 polymorphism has been reported [7]. However, changes in MRP2 activity in vivo is usually associated 
with profound changes in liver exposure, and minor/no effect on plasma exposure, and therefore it usually remains undetected [8]. Recently, it was shown that atorvastatin liver concentration increases 1.64-fold, in the presence of metformin, an Mrp2 inhibitor, without affecting the plasma exposure in Sprague Dawley (SD) rats [9].

Interactions with MRP2 are commonly characterized in vitro, in an inverted membrane vesicle expressing MRP2/Mrp2 in the presence of ATP. Decrease or increase in ATP-dependent transport of probe substrates in the presence of an interacting compound suggest either inhibition or stimulation. To investigate if a compound is an inhibitor of MRP2/Mrp2, estradiol 17- $\beta$-glucuronide (E17 $\beta G)$ is widely used as a probe substrate [1]. However, many compounds such as indomethacin, diclofenac, and benzbromarone are reported to "modulate" the transport of E17 $\beta$ G [10-13]. Modulation refers to stimulation at lower concentrations of the compound, followed by inhibition at higher concentration, providing a "bell-shaped" curve in a percent (\%) transport against interacting compound concentration plots. The compounds that impart similar profile to the transport of probe substrates are henceforth termed as modulators or stimulators interchangeably in this article. The presence of a modulation site, in addition to active transport site in the MRP2 protein, is proposed to explain the modulation and "bell-shaped" curve $[10,14,15]$. There are two manuscripts that explored the in vivo relevance of the in vitro stimulation of Mrp2, in rats. Heredi-Szabo et al. used indomethacin as a stimulator and reported that indomethacin modestly decreases the half-life (40\%) and increases E17 $\beta$ G biliary clearance [16]. In addition, Ito et al. used benzyl penicillin as a stimulator and found that biliary clearance of glutathione (GSH) and bile flow were transiently enhanced (about four-fold for GSH and two-fold for bile flow) [17]. However, in vivo relevance of the substrate-dependence of in vitro MRP2 stimulation remains unknown. In addition, there are no reports of any clinical drug-drug interaction or toxicity that has been attributed to this stimulation. This raises questions on the in vivo relevance of in vitro stimulation of MRP2-mediated probe substrate transport.

Recently, we demonstrated that coproporphyrin-I (CP-I) is an optimal probe substrate for MRP2 [10]. CP-I is a byproduct of heme biosynthesis and its physiological role is not yet clear. The uptake of CP-I in MRP2-overexpressing membrane vesicles follows a typical Michaelis-Menten equation with a low $K_{m}$ value of $7.7 \mu \mathrm{M}$. When CP-I is used as a MRP2 probe for in vitro characterization of MRP2 inhibition, a lower percentage of compounds were found to stimulate MRP2 mediated transport, compared to E17ßG [10]. In our study using 97 compounds, we found $51(53 \%)$ to stimulate MRP2-mediated E17ßG transport, while only 8 out of 47 (17\%) compounds stimulated the transport of CP-I, while 30 compounds were found to inhibit CP-I transport [10]. The results suggest that the stimulation appeared to be a probe substrate-dependent phenomenon, rather than an intrinsic property of the transporter. Furthermore, the stimulators shared the basic backbone structures of physiological steroids, which raises a question of potential in vivo physiological relevance of in vitro stimulation of MRP2-mediated CP-I transport [10].

The aim of the current study was to investigate whether the compounds that stimulated Mrp2-mediated CP-I transport in vitro, also stimulate Mrp2-mediated CP-I transport in vivo. Mrp2-mediated in vivo transport is appreciated by measuring biliary clearance of CP-I and its isomer $\mathrm{CP}-\mathrm{III}$ in rats, in the presence of selected stimulators. The stimulation of CP-I transport in rat Mrp2 transfected membrane vesicles was confirmed and a mechanistic understanding for the perceived in vitro to in vivo disconnect put forward with potential in vitro evidence.

\section{Materials and Methods}

\subsection{Materials/Chemicals}

Mitoxantrone hydrochloride, coproporphyrin-I dihydrochloride (CP-I), progesterone, verapamil, ritonavir and testosterone were procured from Sigma-Aldrich (Sigma-Aldrich Chemie GmbH, Munich, Germany). $\mathrm{d}^{4}$-CP-I (15N $\mathrm{N}^{4}$ deuterated) sodium bisulfate salt procured from Toronto Research Chemicals (Toronto, ON, Canada). MultiScreen Solvinert filter plates $(0.45 \mu \mathrm{m}$, low binding hydrophilic 
polytetrafluoroethylene) were purchased from Millipore (Tullagreen, Ireland). High-performance liquid chromatography-grade methanol was purchased from Merck (Mumbai, India). Formic acid was purchased from Fluka (Fluka Chemie, $\mathrm{GmbH}$, Munich, Germany) and Milli-Q water from Milli-Q system (Millipore SAS, Molsheim, France). Polyethylene-10 and -50 tubing was purchased from Smiths Medical (ASD incorporation, Dublin, OH, USA), and 22-gauge needles and syringes were purchased from Becton Dickinson India Pvt Ltd. (Bangalore, India). Rat Mrp2-expressing inside-out membrane vesicles (protein concentration $4 \mathrm{mg} / \mathrm{mL}$ ) derived from Sf9 insect cells were in-house prepared. Reaction incubation plates (96-well, ultra-low attachment, polystyrene, flat bottom, clear), Filter plate, 96 well ( 0.25 mm Glass Fiber $/ 1.2 \mu \mathrm{m}$ PES) and assay plates (96 well, black, flat bottom, polystyrene) for fluorescence measurement purchased from Corning ${ }^{\circledR} \operatorname{Costar}^{\circledR}$ (Kennebunk, ME, USA). Krebs-Henseleit buffer purchased from Bioreclamation IVT (Bangalore, India). Stock solutions were prepared in dimethyl sulphoxide and stored at $-70^{\circ} \mathrm{C}$.

\subsection{Animals}

Male Sprague Dawley rats weighing 300 to $350 \mathrm{~g}$ (10-12 weeks of age) were obtained from Vivo Bio Tech Ltd., Hyderabad (India). All animal experiments were conducted in the animal research facility of Syngene International Ltd., Bangalore, India, after obtaining approval of the Institutional Animal Ethics Committee (Approval details: Title: Investigation of Metabolism and Elimination pathways of New Chemical Entities (NCEs) in rats; SYNGENE/IAEC/858/07-2017; Principal Investigator/Research Scholar: Mr. Vishwanath K M). The committee was registered for the Purpose of Control and Supervision on Experiments on Animals and accredited by Association for Assessment and Accreditation of Laboratory Animal Care International. The animals were fed a standard laboratory rodent diet (Tetragon Chemie Pvt. Ltd., Bangalore, India) and housed at room temperature $\left(22 \pm 3{ }^{\circ} \mathrm{C}\right)$ and relative humidity of $50 \pm 20 \%$ on a 12-h light and dark cycle. Water was provided ad libitum throughout the study. An intravenous solution formulation of mitoxantrone ( 6 and $15 \mathrm{mg} / \mathrm{mL}$ ) and verapamil $(10 \mathrm{mg} / \mathrm{mL}$ ) were formulated as a solution by using $100 \%$ saline. Progesterone $(1 \mathrm{mg} / \mathrm{mL})$ was formulated using a solution of dimethylacetamide: Solutol: PEG400: water $(5: 5: 75: 15 \% v / v)$.

\subsection{Vesicular Transport Assay}

The vesicular transport assay in membrane vesicles overexpressing rat Mrp2 was conducted using the methods reported previously [10]. Briefly, rat Mrp2 membrane vesicles were diluted to an appropriate concentration in buffer-A containing $50 \mathrm{mM}$ MOPS-Tris (pH 7.0), $70 \mathrm{mM} \mathrm{KCl}, 7.5 \mathrm{mM}$ $\mathrm{MgCl}_{2}$. Membrane vesicles ( $20 \mu \mathrm{L}, 50 \mu \mathrm{g}$ protein) were co-incubated with $0.5 \mu \mathrm{L}$ of test substrates (CP-I) at $37^{\circ} \mathrm{C}$ for $3 \mathrm{~min}$. Then, the reaction was initiated by adding pre-warmed buffer-A $(29.5 \mu \mathrm{L})$ premixed with $4 \mathrm{mM} \mathrm{MgATP}$ or $4 \mathrm{mM} \mathrm{MgAMP}$ and $2 \mathrm{mM}$ glutathione. Following incubation for designated times at $37^{\circ} \mathrm{C}$ on a rotary shaker (Innova 40, New Brunswick Scientific Co., Inc., Enfield, CT, USA) at $100 \mathrm{rpm}$, the reaction was stopped by adding $150 \mu \mathrm{L}$ of cold wash with buffer-B containing $40 \mathrm{mM}$ MOPS-Tris ( $\mathrm{pH} 7.0), 70 \mathrm{mM} \mathrm{KCl}$. The reaction mixture was then transferred into a pre-wet, 96 -well filter plate which was placed onto a filtration device (FiltrEX ${ }^{\mathrm{TM}}$ 96-Well Filter Plates, Corning Technologies India Pvt Ltd., Pune, India), and filtered rapidly via a connected vacuum pump (MultiScreen ${ }^{\circledR} \mathrm{HTS}$ Vacuum, Manifold, MA, USA). All wells were washed 5 times, each time with $200 \mu \mathrm{L}$ of ice-cold wash buffer to remove excess CP-I. After the final wash, the filter plate was dried at room temperature for $1 \mathrm{~h}$. Membrane vesicles were digested using an extraction solvent $(100 \mu \mathrm{L}$ of $0.5 \%$ SDS dissolved in milliQ water, and the plate was kept on a microplate shaker (VWR, Radnor, PA, USA) for 15 min at $230 \mathrm{rpm}$. Then, the filter plate was centrifuged (Eppendorf, Hauppauge, NY, USA) for $2 \mathrm{~min}$ at $2000 \mathrm{rpm}$ along with a receiver plate attached to collect the filtrate. The filtrate was further used to quantify the CP-I levels using a fluorimeter. Fluorescence measurements were conducted with a microplate reader (SpectraMax ${ }^{\circledR}$ M2e, molecular device, San Jose, CA, USA), using 401 and $595 \mathrm{~nm}$ as excitation and emission wavelengths, respectively. ATP-dependent net transport was calculated by 
subtracting the AMP values from those of ATP values. All experiments related to CP-I were conducted in reduced light to minimize florescent bleaching.

Mitoxantrone, testosterone, progesterone, and verapamil were selected to assess their modulatory effects on rat Mrp2-mediated CP-I transport in membrane vesicles. The final assay concentration of CP-I was $5 \mu \mathrm{M}$. The concentrations tested for each modulator were $1,10,50,100,250,500$, and $1000 \mu \mathrm{M}$, except verapamil $(25,50$, and $100 \mu \mathrm{M})$.

\subsection{In Vivo Studies with Bile Duct Cannulated Rats}

Bile duct cannulation on Sprague-Dawley (SD) rats was conducted [18] in a cross-over study design. The freely moving bile duct cannulated rats were kept in metabolic cages. Following $48 \mathrm{~h}$ acclimatization, rats were dosed with saline (dosed in all the groups as a control at $1 \mathrm{~mL} / \mathrm{kg}$ dose volume) and bile, then plasma and urine were collected at specified time points. Ten minutes after the last bile, plasma, and urine sample collection, the same rats were dosed with mitoxantrone, verapamil, and progesterone via the jugular vein as an intravenous infusion at $1 \mathrm{~mL} / \mathrm{kg}$ dose volume by using a single syringe model ' 11 ' Pico plus pump. The doses used were 6 and $15 \mathrm{mg} / \mathrm{kg}$ for mitoxantrone, $10 \mathrm{mg} / \mathrm{kg}$ for verapamil, and $1 \mathrm{mg} / \mathrm{kg}$ for progesterone. The blood samples $(200 \mu \mathrm{L})$ were collected at $0.17,0.25,0.5,0.75,1,1.25,1.5,2,2.5$, and $3 \mathrm{~h}$ and bile samples were collected in pre-weighed amber color tubes over $15 \mathrm{~min}$ intervals up to $1.5 \mathrm{~h}$ and over $30 \mathrm{~min}$ intervals up to $3 \mathrm{~h}$ after dosing mitoxantrone. The blood samples $(200 \mu \mathrm{L})$ were collected at $0.17,0.25,0.5,0.75,1,1.5$, and $2 \mathrm{~h}$ and bile samples were collected in pre-weighed amber color tubes over 10 to $15 \mathrm{~min}$ intervals up to $1 \mathrm{~h}$, and over $30 \mathrm{~min}$ intervals up to $2 \mathrm{~h}$ after dosing verapamil and progesterone. In addition, $0-3 \mathrm{~h}$ urine samples and terminal liver samples were collected from 3 rats using serial sampling. Blood was collected through the jugular vein in tubes containing $2 \% w / v$ potassium ethylenediaminetetraacetic acid solution, and bile was collected in pre-weighed amber tubes, rats were euthanized using carbon dioxide inhalation, liver samples were harvested and homogenized using 4 volumes of water. The bile, plasma, and liver homogenate samples were stored at $-80^{\circ} \mathrm{C}$ until further analysis.

\subsection{Liver Free Fraction Determination for Mitoxantrone, Verapamil and Progesterone}

The free fraction of the test compounds (Mitoxantrone, Verapamil, and Progesterone) in liver was determined using a rapid equilibrium dialysis (RED) device. Ritonavir spiked in human plasma at $2 \mu \mathrm{M}$ concentration was used as a positive control for this purpose. The fu (free fraction) was calculated using the formula given by Riccardi et al. [19]. Briefly, blank liver homogenates were prepared at two different set of dilutions (50-times and 100-times) in $100 \mathrm{mM}$ phosphate buffer using homogenizer (POLYTRON ${ }^{\circledR}$, PT4000, Kinematica AG, Luzern, Switzerland). The test compounds were then individually spiked into these liver homogenates to achieve a target concentration of $10 \mu \mathrm{M}$. RED device (Thermo Fisher Scientific, Hudson, NH, USA) was set up as per the manufacturer's instructions (Instructions, RED Device inserts, 89809, Thermo Scientific, Marietta, OH, USA). An aliquot of $200 \mu \mathrm{L}$ spiked samples were added into the donor side in triplicate, and $350 \mu \mathrm{L}$ of $100 \mathrm{mM}$ potassium phosphate buffer into the respective receiver side and allowed to equilibrate for $6 \mathrm{~h}$ in a HEPA class 100, $\mathrm{CO}_{2}$ incubator (Steri-cycle, Model 370, Thermo Scientific, Marietta, OH, USA) maintained at $37^{\circ} \mathrm{C}$ with $5 \% \mathrm{CO}_{2}$. At the end of the incubation, $25 \mu \mathrm{L}$ incubate from the donor side was aspirated and mixed with $25 \mu \mathrm{L}$ of $100 \mathrm{mM}$ phosphate buffer; similarly, $25 \mu \mathrm{L}$ from receiver side was mixed with $25 \mu \mathrm{L}$ of respective control liver homogenates in a collection plate. Then $150 \mu \mathrm{L}$ of acetonitrile containing internal standard (Propranolol at $150 \mathrm{nM}$ ) was added to the plate and mixed with a multi-channel pipette (Thermo Scientific, FINNPIPETTE F, MH-14040, and Finland). The contents were then transferred to Solvinert filter plate (Merck Millipore Ltd., Tullagreen, Ireland) fitted with $1 \mathrm{~mL}$ capacity 96-well collection plate (Waters Corporation, Milford, MA, USA). The plate was centrifuged at $3220 \times g$ for $5 \mathrm{~min}$ and the filtrate was injected on LC-MS/MS. Analyte concentrations in study samples were then obtained from the calibration standards prepared similarly, and by plotting the analyte to internal standard peak area ratios against respective analyte concentrations. Analyst version 
1.6.2 was used for system control and data processing. The reported \% fu was calculated as average of fu obtained from both 50-times and 100-times dilutions.

\subsection{LC-MS/MS Method}

LC-MS/MS method was developed with adequate sensitivity for the quantification of CP-I, CP-III, mitoxantrone, verapamil, and progesterone using a UPLC system (Waters Corporation, Milford, MA, USA) coupled with a Triple Quad 5500 System (AB Sciex, Framingham, MA, USA), operated in positive electrospray ionization. A combined internal standard solution consisting of $\mathrm{d}^{4}-\mathrm{CP}-\mathrm{I}\left(15 \mathrm{~N}^{4}\right.$ deuterated) sodium bisulfate salt and ritonavir was used during the analysis. $\mathrm{d}^{4}-\mathrm{CP}-\mathrm{I}$ was used as internal standard in the analysis of CP-I and CP-III, whereas ritonavir was used as internal standard (IS) for the analysis of progesterone, mitoxantrone, and verapamil. The analytes and internal standards were separated on an Ace excel 2 C18-150 $\times 2.1 \mathrm{~mm}$ column (Advanced Chromatography Technologies Ltd., Aberdeen, Scotland) using a binary gradient elution with $10 \mathrm{mM}$ ammonium formate in water with $0.1 \%$ formic acid (solvent-A); and acetonitrile with $0.1 \%$ formic acid (solvent-B). The gradient started with $40 \%$ solvent B and maintained for $0.5 \mathrm{~min}$; in $2.5 \mathrm{~min}$, solvent B was increased from 40 to $55 \%$ and then to $95 \%$ in $2.8 \mathrm{~min}$ and remained constant at $95 \%$ solvent B for $1.10 \mathrm{~min}$. Then in 3.80 min solvent B was decreased from 95 to $40 \%$, and it remained constant for 0.2 min, accounting to a total gradient time of $4 \mathrm{~min}$. The flow rate was set at $0.5 \mathrm{~mL} / \mathrm{min}$. The mass spectrometric conditions adopted in the analysis were as follows: Capillary voltage, $5500 \mathrm{~V}$; drying gas temperature, $450{ }^{\circ} \mathrm{C}$; and nebulizer gas pressure, 50 psi (both nebulizer and drying gas were high-purity nitrogen). The MRMs monitored for the compounds were as follows: CP-I (655.5 $\rightarrow 596.4), \mathrm{CP}-\mathrm{III}(655.5 \rightarrow$ $596.4)$, mitoxantrone $(455.1 \rightarrow 358.3)$, verapamil $(455.2 \rightarrow 165.1)$, progesterone $(315.3 \rightarrow 297.5)$, d4-CP-I $(659.3 \rightarrow 600.3)$, and ritonavir $(721.2 \rightarrow 296.2)$. Respective analyte concentrations in study samples were obtained from the calibration curves, plotted as analyte to internal standard peak area ratios against respective analyte concentrations. Analyst version 1.6.2 was used for system control and data processing.

A total of 15 calibration standards were prepared in $1 \%$ bovine serum albumin (BSA) by serial dilution. All the bile samples and urine samples were diluted in 1\% BSA ( 10 fold) prior to analysis. Then $100 \mu \mathrm{L}$ of the sample was quenched with $800 \mu \mathrm{L}$ of acidified acetonitrile (1\% formic acid) containing IS. The samples were vortexed on a spinix mixer (The Scientific House, Chennai, India), and centrifuged in a refrigerated centrifuge (Minispin plus, Eppendorf AG-22331, Hamburg, Germany) at $14,000 \times g, 4{ }^{\circ} \mathrm{C}$ for $5 \mathrm{~min}$. A supernatant of approximately $150 \mu \mathrm{L}$ was transferred into a $96-$ well plate and subjected to LC-MS/MS analysis.

\subsection{Pharmacokinetic Analysis}

CP-I and CP-III area under the plasma concentration-time profiles (AUC) were calculated by non-compartmental analysis and mixed log linear method using Kinetica software (Version 5.5.1; Thermo Electron Corporation, Waltham, MA, USA). Cumulative amounts of CP-I and CP-III were calculated by using Microsoft excel. The biliary (CLb) and renal (CLr) clearances of CP-I and CP-III were estimated by the following equations:

$$
\begin{aligned}
& \mathrm{CL}_{\mathrm{b}}=\frac{\mathrm{X}_{\mathrm{b}}}{\mathrm{AUC}} \\
& \mathrm{CL}_{\mathrm{r}}=\frac{\mathrm{X}_{\mathrm{r}}}{\mathrm{AUC}}
\end{aligned}
$$

where, $X_{b}$ and $X_{r}$ are cumulative amounts of CP-I and CP-III excreted in bile and urine, respectively.

\subsection{Statistical Analysis}

Statistical analysis was performed using the GraphPad Prism program version 5.02 (GraphPad software, San Diego, CA, USA). Mean and standard deviations are calculated in Microsoft Excel 
2013. Student $t$ test was conducted to compare the cumulative amount of CP-I and CP-III obtained at each time point when administered saline and different modulators (mitoxantrone, verapamil, and progesterone); $p<0.05$ was regarded as significant. No statistical test was conducted in in vitro experiments, as they represent triplicate data from only one experiment.

\section{Results}

\subsection{Mrp2-Mediated CP-I Transport in Membrane Vesicles}

In time linearity studies, Mrp2-mediated CP-I transport was linear with time up to $30 \mathrm{~min}$, and a 15 min time point was used for all incubations unless specified. The transport kinetics of CP-I were then characterized by assessing concentration-dependent transport of CP-I in membrane vesicles overexpressing rat Mrp2 protein. ATP over AMP ratio of CP-I uptake is 23, with negligible uptake in the presence of AMP. As depicted in Figure 1, the concentration-dependent CP-I uptake appeared to follow a hyperbolic relationship. The kinetic curve could be adequately modelled by the standard Michaelis-Menten equation. The apparent $K_{m}$ and $V_{\max }$ values (mean \pm SD) for CP-I were $15 \pm 6 \mu \mathrm{M}$ and $161 \pm 20 \mathrm{pmol} / \mathrm{min} / \mathrm{mg}$ protein, respectively.

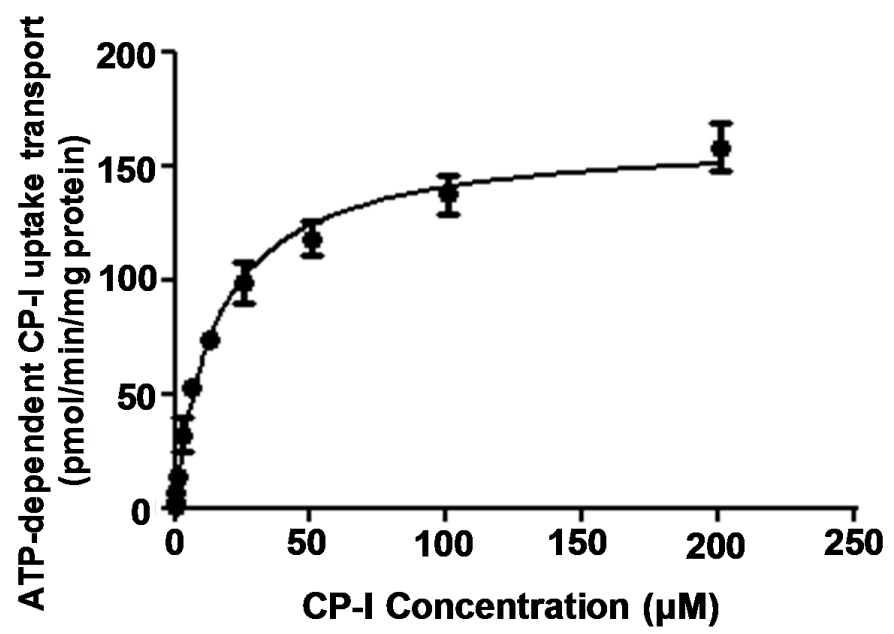

Figure 1. Concentration-dependent uptake transport of coproporphyrin-I (CP-I) in rat multidrug resistance-associated protein 2 (Mrp2) membrane vesicles. Rat Mrp2 membrane vesicles were incubated with CP-I at $37^{\circ} \mathrm{C}$ for $15 \mathrm{~min}$. ATP-dependent CP-I uptake is measured by subtracting the uptake in the presence of AMP from that of ATP. For more experimental details refer to Section 2.3. All data values are presented as mean and SD of a single experiment performed in triplicate wells.

Figure 2 shows the concentration-dependent effect of various modulators (mitoxantrone, progesterone, testosterone, and verapamil) on the uptake of CP-I in rat Mrp2-overexpressing membrane vesicles. These modulators were selected based on the previous results found with human MRP2 vesicles [10]. As expected, all four modulators showed increased uptake of CP-I (stimulation) up to a specific concentrations and then showed inhibition of uptake at higher concentrations in rat Mrp2 vesicles, leading to a bell-shaped curve. This trend of stimulation and inhibition is common for all the four modulators, although at different concentration ranges, which agree with the results observed in human MRP2 vesicles (Gilibili et al., 2017). The interactions between CP-I uptake in rat Mrp2 vesicles and the above stimulators were further conducted in the presence of $10 \mu \mathrm{M}$ bilirubin. As shown in Figure 3, the presence of bilirubin suppressed the stimulation of CP-I transport in rat Mrp2 vesicles by $200 \mu \mathrm{M}$ mitoxantrone, $100 \mu \mathrm{M}$ progesterone, and $100 \mu \mathrm{M}$ verapamil. The suppression reached a maximum (about $50 \%$ ) at $100 \mu \mathrm{M}$ mitoxantrone. 


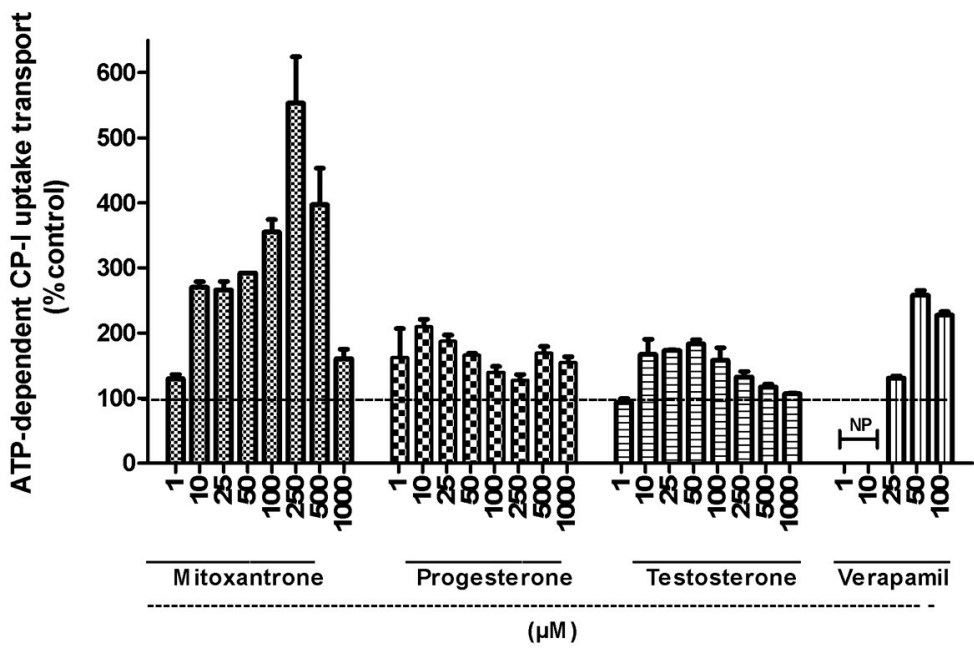

Figure 2. Effect of various modulators (mitoxantrone, progesterone, testosterone, and verapamil) on the uptake of CP-I in rat Mrp2 membrane vesicles. Rat Mrp2-mediated CP-I transport was measured in inside-out membrane vesicles. Mrp2 vesicle protein $(50 \mu \mathrm{g} /$ well $)$ were preincubated with modulators at above reported concentrations at $37^{\circ} \mathrm{C}$ and reaction was started by adding CP-I ( $\left.5 \mu \mathrm{M}\right)$ and incubated for $15 \mathrm{~min}$. NP; not performed. For more on experimental details refer to Section 2.3. Dotted line represents basal level (100\%) of CP-I uptake in control wells in the presence of ATP with no modulators added. All data values presented as mean and SD of single experiment performed in triplicate wells. In the inset, same data set is represented in curve to show the bell shape of the concentration-dependent stimulation and inhibition (also referred as modulation in the text).

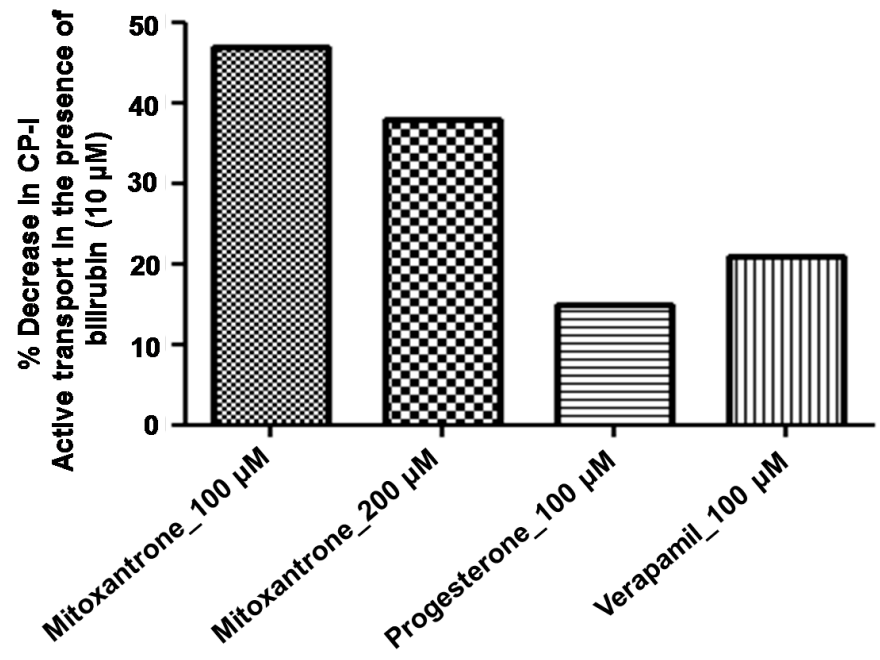

Figure 3. Effect of bilirubin on the transport of CP-I using rat Mrp2 vesicles in the presence and absence of modulators. Rat Mrp2 vesicles $(50 \mu \mathrm{g} /$ well) were preincubated with and without bilirubin $(10 \mu \mathrm{M})$ and modulators for $3 \mathrm{~min}$ at $37^{\circ} \mathrm{C}$. \% Net decrease in ATP-dependent CP-I uptake in presence of bilirubin was calculated by taking CP-I uptake values in presence of modulators as control. All data values presented as mean of single experiment performed in duplicate wells. The duplicate values are: $54 \%$, and $40 \%$ (mitoxantrone $100 \mu \mathrm{M}$ ), 34\% and $42 \%$ (mitoxantrone $200 \mu \mathrm{M}$ ), $17 \%$ and $12 \%$ (progesterone $100 \mu \mathrm{M}$ ), and 21, and 21\% (verapamil $100 \mu \mathrm{M}$ ).

\subsection{Plasma Level and Biliary Secretion of Endogenous CP-I and CP-III in Rats}

To understand whether the observed in vitro effect of modulators on transport of CP-I is translated in in vivo systems, studies were conducted using bile duct cannulated rats. In this model, the modulators were administered as an infusion through the jugular vein. Plasma and bile levels of 
CP-I and CP-III were measured at different time intervals during $2 \mathrm{~h}$ (for verapamil and progesterone) and $3 \mathrm{~h}$ (for mitoxantrone) pre- and post-dose treatment with modulators. The basal levels of CP-I and CP-III excreted in $2 \mathrm{~h}$ were found to be about 600 and 1200 pmoles in rat bile, respectively. The plasma AUCs (0-2 h) were found to be about 1.2 and 9.5 nM.h for CP-I and CP-III, respectively. Figure 4 shows the amount CP-I and -III excreted in bile, in the presence and absence of the modulators at different time durations. As can be observed from Figure 4, there was a significant increase in biliary excretion of CP-I and III between 0.25 and $0.5 \mathrm{~h}$ following dosing of mitoxantrone $6 \mathrm{mg} / \mathrm{kg}$ (Figure 4A1,D1). However, the same changes were not observed with $15 \mathrm{mg} / \mathrm{kg}$ mitoxantrone (Figure 4A2,D2). At the higher dose of mitoxantrone $(15 \mathrm{mg} / \mathrm{kg})$, the biliary secretion of CP-I was decreased from 0.33 to $1.25 \mathrm{~h}$. In contrast, with administration of progesterone at $1 \mathrm{mg} / \mathrm{kg}$, a significant decrease in biliary secretion of CP-III from 0.17 to $1.5 \mathrm{~h}$ was observed. A similar decrease of CP-I biliary clearance was also observed from 0 to $0.75 \mathrm{~h}$, following $10 \mathrm{mg} / \mathrm{kg}$ verapamil treatment.

\section{Coproporphyrin-I}

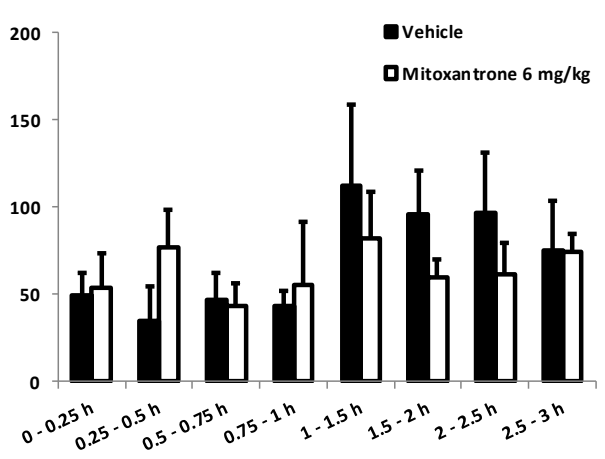

(A1)

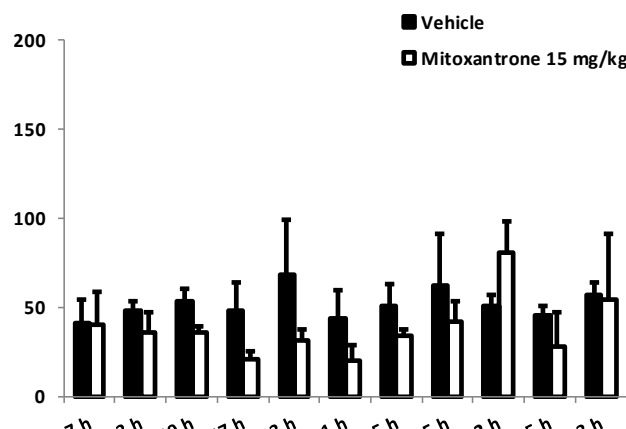

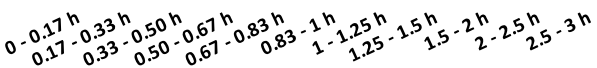

(A2)

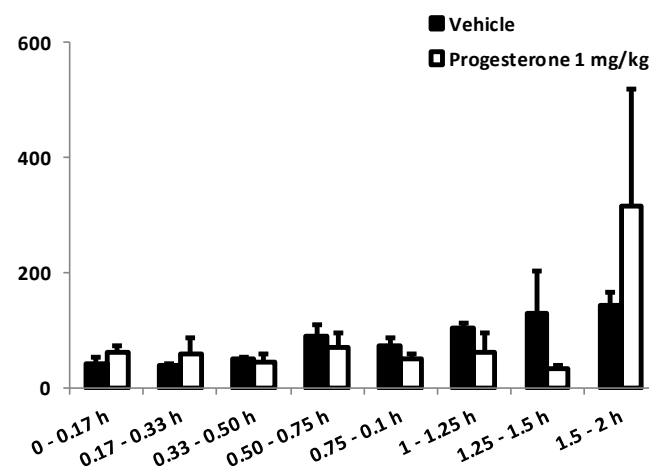

(B)

\section{Coproporphyrin-III}

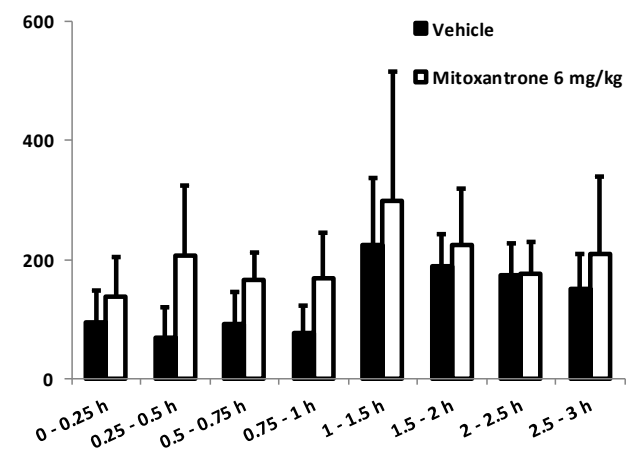

(D1)

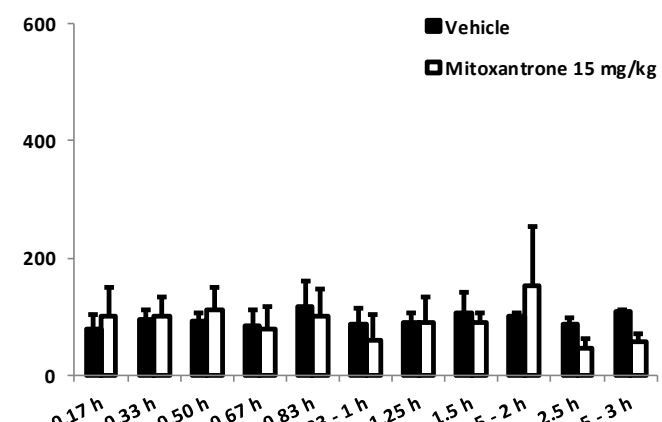

(D2)

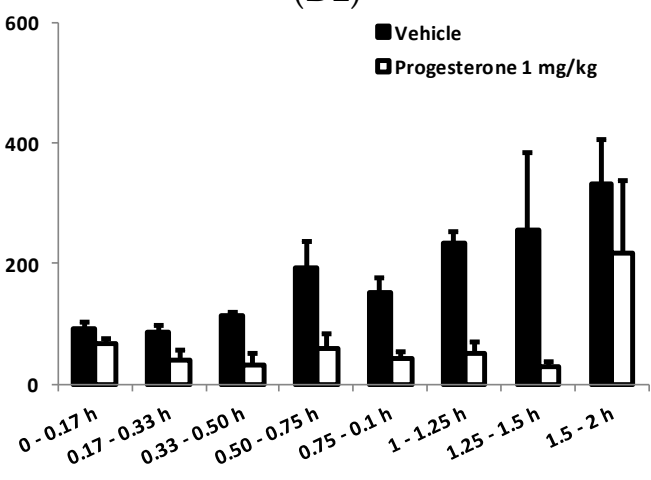

(E)

Figure 4. Cont. 


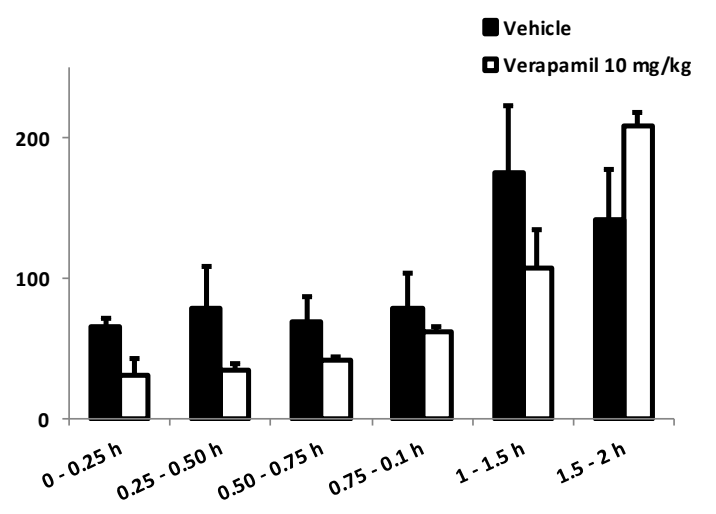

(C)

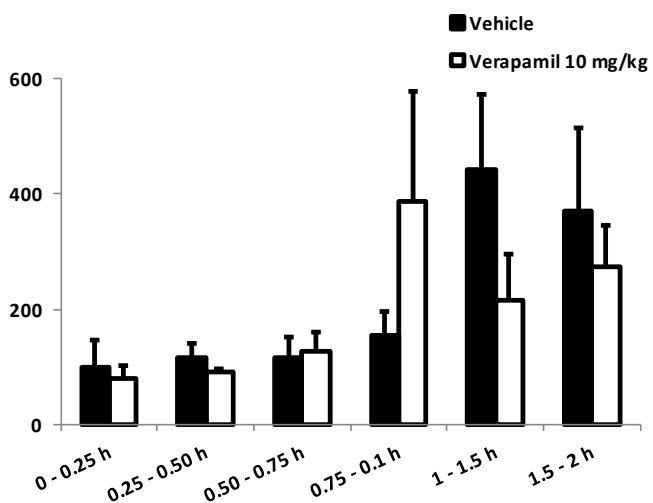

(F)

\section{Time interval}

Figure 4. Amount of CP-I and CP-III excreted in bile at different time intervals in the presence ( $\square$ ) and absence ( $\mathbf{\square})$ of modulators. Biliary excretion profile of endogenous CP-I and CP-III in male Sprague Dawley (SD) rats following intravenous administration of various modulators. All animals received saline $(0.9 \% \mathrm{NaCl})$ first then modulator after collection of bile at designated time points. (A1,D1,A2,D2); represent biliary excretion profile of $\mathrm{CP}-\mathrm{I}$ and $\mathrm{CP}-\mathrm{III}$ in male SD rats treated with mitoxantrone at 6 and $15 \mathrm{mg} / \mathrm{kg}$, respectively. (B,E) represents biliary excretion profile of CP-I and CP-III in male SD rats treated with progesterone at $1 \mathrm{mg} / \mathrm{kg}$. (C,F) represents biliary excretion profile of CP-I and CP-III in male SD rats treated with verapamil at $10 \mathrm{mg} / \mathrm{kg}$. All the data values presented in mean and SD of single experiment performed in three animals.

The biliary (Table 1) and renal clearances (Supplementary Table S2) over the 2 or $3 \mathrm{~h}$ time periods, with and without the modulators treatment, were calculated as described in materials and methods. The average biliary clearance of CP-I is four-fold higher than CP-III in the control experiments, while urinary clearance of CP-III is three-fold higher than CP-I. No significant increase in biliary or urinary clearance of CP-I or CP-III is observed at pre- and post-dosing with modulators $(p>0.05)$. Supplementary Table S1 lists the cumulative amount of CP-I and CP-III excreted in bile, in the 2 or $3 \mathrm{~h}$ time interval. The cumulative amount of CP-I excreted in bile and the plasma AUC of CP-I were found to be similar in the presence and absence of all the three modulators. A similar trend was also observed in CP-III levels.

Table 1. Biliary clearances coproporphyrin-I (CP-I) and CP-III in male Sprague Dawley (SD) rats treated with vehicle or modulator.

\begin{tabular}{cccc}
\hline \multirow{2}{*}{ Dose Group } & \multirow{2}{*}{ Modulator Dose $(\mathbf{m g} / \mathbf{k g}, \mathbf{i . v})$} & \multicolumn{2}{c}{ Biliary Clearance ${ }^{\#}$ (mL/min) } \\
\cline { 3 - 4 } & & CP-I & CP-III \\
\hline Vehicle & - & $7.6 \pm 1.5$ & $2.3 \pm 1.3$ \\
Mitoxantrone & 6 & $5.9 \pm 0.3$ & $1.9 \pm 1.7$ \\
Vehicle & - & $11 \pm 0.1$ & $3.3 \pm 0.03$ \\
Mitoxantrone & 15 & $16 \pm 9.5$ & $3.4 \pm 2.1$ \\
Vehicle & - & $14 \pm 6.0$ & $2.3 \pm 0.2$ \\
Progesterone & 1 & $11 \pm 4.6$ & $2.3 \pm 0.3$ \\
Vehicle & - & $5.8 \pm 2.1$ & $1.6 \pm 0.5$ \\
Verapamil & 10 & $3.8 \pm 0.8$ & $1.2 \pm 0.6$ \\
\hline
\end{tabular}

\#: All data values are presented in Mean $\pm \mathrm{SD}$, collected from three animal. For experimental design refer to Section 2.4. Saline $(0.9 \% \mathrm{NaCl})$ was used as vehicle. 
The total and free liver concentrations of the stimulators followed by i.v infusion were determined. The liver concentrations of mitoxantrone (at $15 \mathrm{mg} / \mathrm{kg}$ ), progesterone, and verapamil were 950, 197, and $1040 \mu \mathrm{M}$, respectively, resulting in free liver concentration $\left(\mathrm{C}_{\max }\right.$, unbound, liver) of mitoxantrone, progesterone, and verapamil $0.28,1.86$, and $25.8 \mu \mathrm{M}$, respectively (Table 2).

Table 2. Liver unbound concentrations of modulators.

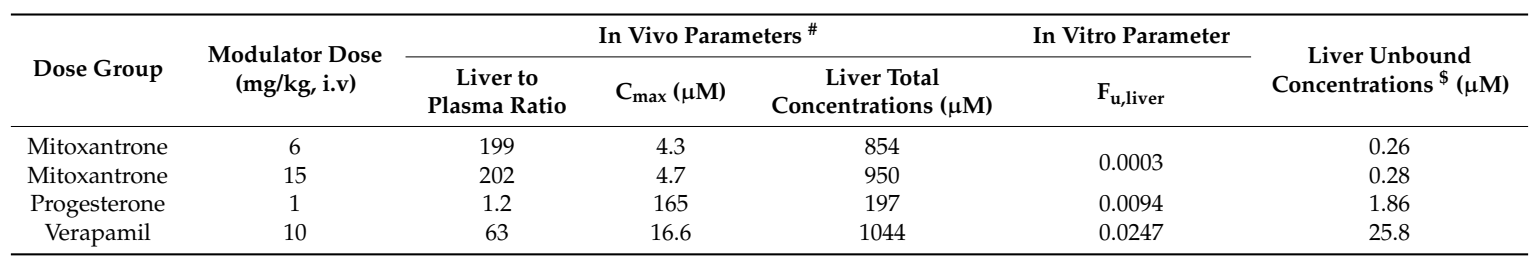

\#: Livers were collected at the terminal time point ( 2 or $3 \mathrm{~h}$ ) of the respective study, and modulator concentrations were determined in liver homogenate by LC-MS/MS method, refer to Section 2.5 for more details. $\mathrm{F}_{\mathrm{u} \text {,iver: modulator }}$ free fraction in liver was determined using rapid equilibrium dialysis method, using blank liver homogenate as matrices. ${ }^{\$}$ : Liver unbound concentrations were determined by multiplying $\mathrm{F}_{\mathrm{u} \text {,liver }}$ with total liver concentrations.

\section{Discussion}

In vitro stimulation of drug metabolizing enzymes and transporters has long been recognized. However, in vivo or clinical impact of stimulation of drug metabolizing enzymes or transporters are scarce $[20,21]$. Therefore, the translation of in vitro stimulation to in vivo has not been fully understood. For example, a two-fold stimulation of flurbiprofen metabolism in liver microsome in presence of dapsone yields only $10 \%$ increase in flubiprofen clearance in humans, which appears not to be clinically relevant [22,23]. In vitro stimulation of MRP2-mediated transport of different probe substrates, in the presence of a chemically diverse set of compounds, have been widely reported $[10,13,14,24]$. Previously, we reported that $53 \%$ compound of a list of 97 compounds stimulated MRP2-mediated E17 $\beta G$ transport [10]. We found that the stimulation is substrate dependent, as lesser percentage $(17 \%)$ of compounds stimulated CP-I transport [10]. So far, two previous reports investigated in vivo stimulation of Mrp2- mediated probe substrate transport by measuring the probe compounds, E17 $\beta \mathrm{G}$ and GSH, respectively, in rat bile, following treatment with stimulators $[16,17]$. However, as previously explained, MRP2/Mrp2-mediated E17 $\beta$ G transport is known to be stimulated by many compounds with diverse chemical motifs. We have previously shown that the stimulators used in the study of Heredi-Szabo et al., benzbromarone and indomethacin, are actually inhibitors of MRP2, when CP-I is used as a probe substrate [10]. Therefore, the stimulation effect was re-investigated with CP-I as endogenous biomarker. CP-I, being endogenous, provided us with the advantage that external administration of a radioactive/labelled probe compound could be avoided.

In agreement with the results found in human MRP2 vesicles, rat Mrp2 mediated CP-I transport also displayed conventional Michaelis-Menten kinetics, with Km of $15 \mu \mathrm{M}$. The Km values of CP-I in rat Mrp2 vesicles were comparable to that in human ( $15 \mathrm{vs.} 7.7 \mu \mathrm{M}$ for rat and human MRP2, respectively), suggesting the interspecies differences in affinity of CP-I towards MRP2/Mrp2-meditated biliary secretion is minimum. This is in contrast to E17 $\beta G$, as significant differences in kinetics of E17 $\beta G$ are reported between different species, such as Michaelis-Menten reported for rat, while sigmoidal kinetics reported for human $[14,16]$. Four stimulators, mitoxantrone, verapamil, testosterone, and progesterone were selected as tool compounds for the rat in vitro and in vivo investigations, based on their significant stimulation of CP-I transport observed in human MRP2 vesicles and the structural features [10]. All four compounds (structures in supplementary Figure S2) stimulated CP-I transport in membrane vesicles overexpressing rat Mrp2, with mitoxantrone being the strongest stimulator. Mitoxantrone showed a bell-shaped curve: stimulation of Mrp2 mediated CP-I transport at lower concentrations (up to $250 \mu \mathrm{M}$ ) followed by inhibition at higher $(>250 \mu \mathrm{M})$ concentrations of mitoxantrone (Figure 2$)$. Therefore, two doses of mitoxantrone $(6 \mathrm{mg} / \mathrm{kg}$ and $15 \mathrm{mg} / \mathrm{kg}$ ) were administered in bile duct cannulated rats, to assess the impact on CP-I biliary clearance. Mitoxantrone, verapamil $(6 \mathrm{mg} / \mathrm{kg})$, and progesterone 
$(1 \mathrm{mg} / \mathrm{kg}$ ) were investigated by a cross-over study design, so that the drug-pretreatment CP-I and CP-III biliary clearance values can be used as controls to compare with post drug-treatment biliary clearances. Collection of bile was initiated at early time points, as previous reports suggest that the stimulation of Mrp2 mediated transported appeared to occur within the first hour of dosing of indomethacin or benzylpenicillin [16,17].

Contrary to in vitro findings, no significant difference in either biliary clearances or cumulative amount of CP-I or CP-III eliminated in bile were detected before and after treatment of mitoxantrone (Table 1 and Figure 4, Supplementary Table S1). Similarly, neither biliary clearance nor cumulative amount of CP-I excreted of in bile were increased, when dosed with progesterone or verapamil. Although there was a significant increase in CP-I and CP-III levels in bile between 15-30 min, following the $6 \mathrm{mg} / \mathrm{kg}$ mitoxantrone dose (Figure 3), the same effect was not observed at the higher dose, nor at following or earlier time intervals in the same dose, suggesting that could be an experimental error. To know if the lack of in vivo translatability of in vitro stimulation is because the compounds are not attaining sufficient concentrations in the liver, free liver concentrations of mitoxantrone, progesterone, and verapamil were determined. In vitro, $1 \mu \mathrm{M}$ progesterone and $25 \mu \mathrm{M}$ verapamil stimulated the Mrp2 mediated transport of CP-I to $>1.5$-fold and $>1.3$-fold, respectively (Figure 2). The free liver exposures obtained following dosing progesterone and verapamil are 1.86 and $25.8 \mu \mathrm{M}$ respectively (Table 2). Hence, we can confidently conclude that even after reaching relevant concentrations in liver, progesterone and verapamil did not stimulate Mrp2-mediated CP-I transport in rats. Mitoxantrone dosing was limited by its observed toxicity in rats. Therefore, we could not reach free liver concentration beyond $0.28 \mu \mathrm{M}$, while in vitro stimulations started from $1 \mu \mathrm{M}$ onwards. In vitro, in presence of vesicles, the "free" concentration of the compound is expected to be lower than $1 \mu \mathrm{M}$, because of compounds binding to vesicles. Thus, 'vesicle binding' will further lower in vitro concentration and we can comfortably assume that "free in vitro concentration" will be in similar range as of "free in vivo concentration".

Furthermore, apart from direct measurement of CP-I in bile, there are two indirect evidences of not observing any in vivo stimulation of Mrp2 function by mitoxantrone, progesterone, and verapamil. First, the bile flow is not enhanced following compound treatment. Glutathione excretion in bile via Mrp2 mediates bile salt-independent bile flow $[25,26]$. We assessed the bile flow before and after treatment with respective stimulators and did not observe any increase in bile flow following treatment with the stimulators (Supplementary Table S3). The second indirect proof comes from urinary coproporphyrin ratio (UCP-I/UCP-(I + III)), which is often used as a surrogate marker of MRP2 function and has been used to assess methotrexate PK variability [27]. The urinary coproporphyrin ratio did not decrease following dosing the stimulators (Supplementary Table S4. Collectively, we concluded that the in vitro stimulation of MRP2/Mrp2-mediated CP-I transport is not translatable in vivo.

Plasma and urinary coproporphyrins (CP-I and III) have been suggested as in vivo and clinical biomarkers for organic anion transporting polypeptide (OATP)-mediated drug interactions [28-30]. The plasma and urinary levels of CPs are shown to be sensitive markers of Oatp-mediated DDI in cynomolgus monkeys and mice [30]. CP-I uptake in isolated rat hepatocytes was shown to be rifamycin sensitive (Supplementary Figure S1), suggesting a similar role of Oatp transporters in CP dispositions among mice, rats, monkeys, and humans [30]. This opens the possibility of using rats as an investigational model to understand CP-I as an in vivo marker for Oatp inhibition.

In addition to hepatocyte canalicular membranes, Mrp2 is also expressed on the apical side of renal proximal tubules. Thus, one can argue that functional activity of Mrp2 can be monitored via urinary levels of CPs. However, other MRP/Mrp isoforms on the apical side of proximal tubule cells (such as Mrp4), with overlapping substrate specificity, can interfere with Mrp2 function estimation via renal secretion of CPs. Therefore, urinary elimination of CPs is not assessed to understand the in vivo impact of stimulation. In the present investigation, we found the basal plasma levels of CP-III are three-four times higher than CP-I levels, which is similar to what is reported in mice, and is in 
contrast to monkeys and humans, where CP-I levels are higher in plasma ([30] and Supplementary Table S1). In addition, the biliary clearance of CP-I is about four-fold higher than CP-III, while the urinary clearance of CP-III is three-fold higher than CP-I. This suggests there can be preferential affinity of CP-I towards biliary transporters while CP-III towards urinary transporters. Higher renal clearance of CP-III, compared to CP-I, is observed for monkeys as well [30].

To further investigate the possible mechanism for the in vitro-in vivo disconnect of stimulation of CP-I transport via Mrp2, the effect of endogenous compounds such as bile acids and bilirubin on CP-I transport were evaluated. Bile acids did not decrease stimulation by the stimulators (data not shown), while $10 \mu \mathrm{M}$ bilirubin suppressed the stimulation of Mrp2-mediated CP-I transport to about $50 \%$ and $30 \%$ of what is observed with 100 and $200 \mu \mathrm{M}$ mitoxantrone, respectively. To a lesser extent, bilirubin also decreased the stimulation of CP-I transport by $100 \mu \mathrm{M}$ progesterone and verapamil. Due to the unavailability of bilirubin glucuronides, we were not be able to further assess its effect on the stimulation of Mrp2-mediated CP-I transport by these stimulators. This is the first time that the effect of bilirubin on the in vitro stimulation of Mrp2-mediated transport is reported, to the best of our knowledge. Thus, we hypothesize that endogenous compounds may act on the postulated modulator or transport site of MRP2/Mrp2 and contribute to the in vitro-in vivo discrepancy. This can add to the previous assumptions that the differences in membrane composition between the in vitro and in vivo systems [31] may lead to perceived in vitro to in vivo disconnect in transporter function. The possibility to add bilirubin in vitro to minimize the stimulation effects on MRP2-mediated transport could be further explored to improve the in vitro tools in assessing MRP2 inhibition. Further investigation with altering the in vitro assay can provide confirmatory data in this regard.

\section{Conclusions}

In this work, we first showed that CP-I follows a Michaelis-menten kinetics in rat Mrp2 vesicles. Few compounds (verapamil, mitoxantrone, progesterone, and testosterone) which stimulated MRP2-mediated CP-I transport in human MRP2 vesicles also stimulated rat Mrp2-mediated CP-I transport in vitro. In order to understand the in vivo significance of the 'stimulation', bile-duct cannulated rats were dosed with mitoxantrone, verapamil, and progesterone, and CP-I and CP-III levels were measured in bile and urine. Different doses of mitoxantrone, verapamil, or progesterone do not impact Mrp2-mediated CP-I and CP-III excretion in rat bile. This was contradictory to the in vitro data, where mitoxantrone, verapamil, and progesterone stimulated CP-I transport mediated by Mrp2. Our findings support non-existence of any clinical event that can be attributed to stimulation of MRP2-mediated transport. In addition, we also provide evidence in favor of the hypotheses that the presence of an endogenous compound such as bilirubin may lead to the in vitro and in vivo discrepancy.

Supplementary Materials: The following are available online at http:/ /www.mdpi.com/1999-4923/10/3/125/s1, Figure S1: CP-I uptake in presence and absence of $1 \mathrm{mM}$ rifamycin SV in suspended rat hepatocytes; Figure S2: Structures of the modulators (mitoxantrone, progesterone, testosterone and verapamil) used in this study, along with conventional probe substrate of MRP/Mrp2, estradiol b-glucuronide.; Table S1: Cumulative amount of excretion and area under plasma concentration-time (AUC) profile of CP-I and CP-III following i.v injection of vehicle or modulator.; Table S2: Renal clearance of CP-I and CP-III following i.v injection of vehicle or modulator.; Table S3: Bile flow rate $(\mathrm{ml} / \mathrm{h})$ before and after treatment with modulators. The cumulative volume is divided by the total time span of bile collection, to obtain bile flow rate; Table S4: Urinary coproporphyrin (UCP-I/(UCP-I+UCP-III)) ratios with vehicle and compound dosing.

Author Contributions: Conceptualization: S.C., Y.L., R.R.G., T.T.M., H.S., V.K.; Methodology: R.R.G., V.K., B.V.M., S.C.; Resources: R.R.G., V.K., B.V.M, S.C.; Data Curation: S.C., R.R.G., T.T.M., V.K., B.V.M.; Writing-original draft preparation: S.C., Y.L., R.R.G., V.K., B.V.M., T.T.M.; Writing-review and editing: S.C., Y.L., H.S.; Footnote: R.R.G. and V.K. equally contributed to the work.

Funding: This research received no external funding. 
Acknowledgments: We would like to thank Punit Marathe, and Mike Sinz during various aspects of this work, e.g., data analysis, experimental set up, reviewing manuscript. We would like to thank Prabhakar Rajanna for helping bioanalysis, Anoop Kumar for providing freshly isolated rat hepatocytes, Ram Vishwanath for assisting in in vivo experiments and Harbeer Kaur for helping in data analysis.

Conflicts of Interest: Authors declare no conflict of interest. S.C., R.R.G., V.K., B.V.M., T.T.M. are working in Biocon Bristol Myers-Squibb Research Center for last 3 years or more. H.S. is working in BMS, NJ for more than 5 years. Y.L. was working in BMS, NJ till 2017, currently he is working in Gilead, Foster City.

\section{Abbreviations}

$\begin{array}{ll}\text { E17 } \beta \text { G } & \text { estradiol-17 } \beta \text {-glucuronide } \\ \text { MRP2 } & \text { multidrug-resistance associated protein } 2 \\ \text { OATP/Oatp } & \begin{array}{l}\text { organic anion transporting polypeptide } \\ \text { coproporphyrin-I } \\ \text { CP-I }\end{array} \\ \text { CP-III } & \text { coproporphyrin-III LC-MS/MS, liquid chromatography-tandem mass spectrometry } \\ \text { ATP } & \text { adenosine mono-phosphate } \\ \text { AMP } & \text { ATP binding cassette } \\ \text { ABC } & \text { glutathione } \\ \text { GSH } & \text { Cholic acid } \\ \text { CA } & \text { Glycocholic acid } \\ \text { GCA } & \text { taurocholic acid } \\ \text { TCA } & \text { drug-drug interactions }\end{array}$

\section{References}

1. Hillgren, K.; Keppler, D.; Zur, A.; Giacomini, K.; Stieger, B.; Cass, C.; Zhang, L. Emerging transporters of clinical importance: An update from the International Transporter Consortium. Clin. Pharmacol. Ther. 2013, 94, 52-63. [CrossRef] [PubMed]

2. Lai, Y.; Hsiao, P. Beyond the ITC White Paper: Emerging sciences in drug transporters and opportunities for drug development. Curr. Pharm. Des. 2014, 20, 1577-1594. [CrossRef] [PubMed]

3. Keppler, D.; Konig, J.; Buchler, M. The canalicular multidrug resistance protein, cMRP/MRP2, a novel conjugate export pump expressed in the apical membrane of hepatocytes. Adv. Enzym. Regul. 1997, 37, 321-333. [CrossRef]

4. Keppler, D.; Leier, I.; Jedlitschky, G. Transport of glutathione conjugates and glucuronides by the multidrug resistance proteins MRP1 and MRP2. Biol. Chem. 1997, 378, 787-791. [PubMed]

5. Borst, P.; Evers, R.; Kool, M.; Wijnholds, J. A family of drug transporters: The multidrug resistance-associated proteins. J. Natl. Cancer Inst. 2000, 92, 1295-1302. [CrossRef] [PubMed]

6. Yoshida, K.; Maeda, K.; Sugiyama, Y. Hepatic and intestinal drug transporters: Prediction of pharmacokinetic effects caused by drug-drug interactions and genetic polymorphisms. Annu. Rev. Pharmacol. Toxicol. 2013, 53, 581-612. [CrossRef] [PubMed]

7. Rau, T.; Erney, B.; Göres, R.; Eschenhagen, T.; Beck, J.; Langer, T. High-dose methotrexate in pediatric acute lymphoblastic leukemia: Impact of ABCC2 polymorphisms on plasma concentrations. Clin. Pharmacol. Ther. 2006, 80, 468-476. [CrossRef] [PubMed]

8. Toshimoto, K.; Tomoda, Y.; Chiba, K.; Sugiyama, Y. Analysis of the change in the blood concentration-time profile caused by complex drug-drug interactions in the liver considering the enterohepatic circulation: Examining whether the inhibition constants for uptake, metabolism, and biliary excretion can be recovered by the analyses using physiologically based pharmacokinetic modeling. J. Pharm. Sci. 2017, 106, 2727-2738. [CrossRef] [PubMed]

9. Shin, E.; Shin, N.; Oh, J.-H.; Lee, Y.-J. High-dose metformin may increase the concentration of atorvastatin in the liver by inhibition of multidrug resistance-associated protein 2. J. Pharm. Sci. 2017, 106, 961-967. [CrossRef] [PubMed]

10. Gilibili, R.R.; Chatterjee, S.; Bagul, P.; Mosure, K.W.; Murali, B.V.; Mariappan, T.T.; Mandlekar, S.; Lai, Y. Coproporphyrin-I: A fluorescent, endogenous optimal probe substrate for ABCC2 (MRP2) suitable for vesicle-based MRP2 inhibition assay. Drug Metab. Dispos. 2017, 45, 604-611. [CrossRef] [PubMed] 
11. Kidron, H.; Wissel, G.; Manevski, N.; Hakli, M.; Ketola, R.A.; Finel, M.; Yliperttula, M.; Xhaard, H.; Urtti, A. Impact of probe compound in MRP2 vesicular transport assays. Eur. J. Pharm. Sci. 2012, 46, 100-105. [CrossRef] [PubMed]

12. Zelcer, N.; Huisman, M.T.; Reid, G.; Wielinga, P.; Breedveld, P.; Kuil, A.; Knipscheer, P.; Schellens, J.H.; Schinkel, A.H.; Borst, P. Evidence for two interacting ligand binding sites in human multidrug resistance protein 2 (ATP binding cassette C2). J. Biol. Chem. 2003, 278, 23538-23544. [CrossRef] [PubMed]

13. Zimmermann, C.; van de Wetering, K.; van de Steeg, E.; Wagenaar, E.; Vens, C.; Schinkel, A.H. Species-dependent transport and modulation properties of human and mouse multidrug resistance protein 2 (MRP2/Mrp2, ABCC2/Abcc2). Drug Metab. Dispos. 2008, 36, 631-640. [CrossRef] [PubMed]

14. Borst, P.; Zelcer, N.; Van De Wetering, K. MRP2 and 3 in health and disease. Cancer Lett. 2006, $234,51-61$. [CrossRef] [PubMed]

15. Hirono, S.; Nakagome, I.; Imai, R.; Maeda, K.; Kusuhara, H.; Sugiyama, Y. Estimation of the three-dimensional pharmacophore of ligands for rat multidrug-resistance-associated protein 2 using ligand-based drug design techniques. Pharm. Res. 2005, 22, 260-269. [CrossRef] [PubMed]

16. Heredi-Szabo, K.; Glavinas, H.; Kis, E.; Mehn, D.; Bathori, G.; Veres, Z.; Kobori, L.; von Richter, O.; Jemnitz, K.; Krajcsi, P. Multidrug resistance protein 2-mediated estradiol-17 $\beta$-D-glucuronide transport potentiation: In vitro-in vivo correlation and species specificity. Drug Metab. Dispos. 2009, 37, 794-801. [CrossRef] [PubMed]

17. Ito, K.; Koresawa, T.; Nakano, K.; Horie, T. Mrp2 is involved in benzylpenicillin-induced choleresis. Am. J. Physiol. Gastroint. Liver Physiol. 2004, 287, G42-G49. [CrossRef] [PubMed]

18. Burden, N.; Kendrick, J.; Knight, L.; McGregor, V.; Murphy, H.; Punler, M.; van Wijk, H. Maximizing the success of bile duct cannulation studies in rats: Recommendations for best practice. Lab. Anim. 2017, 51, 457-464. [CrossRef] [PubMed]

19. Riccardi, K.; Cawley, S.; Yates, P.D.; Chang, C.; Funk, C.; Niosi, M.; Lin, J.; Di, L. Plasma protein binding of challenging compounds. J. Pharm. Sci. 2015, 104, 2627-2636. [CrossRef] [PubMed]

20. Hutzler, J.M.; Tracy, T.S. Atypical kinetic profiles in drug metabolism reactions. Drug Metab. Dispos. 2002, 30, 355-362. [CrossRef] [PubMed]

21. Van Waterschoot, R.A.; Rooswinkel, R.W.; Sparidans, R.W.; van Herwaarden, A.E.; Beijnen, J.H.; Schinkel, A.H. Inhibition and stimulation of intestinal and hepatic CYP3A activity: Studies in humanized CYP3A4 transgenic mice using triazolam. Drug Metab. Dispos. 2009, 37, 2305-2313. [CrossRef] [PubMed]

22. Hutzler, J.M.; Frye, R.F.; Korzekwa, K.R.; Branch, R.A.; Huang, S.-M.; Tracy, T.S. Minimal in vivo activation of CYP2C9-mediated flurbiprofen metabolism by dapsone. Eur. J. Pharm. Sci. 2001, 14, 47-52. [CrossRef]

23. Korzekwa, K.; Krishnamachary, N.; Shou, M.; Ogai, A.; Parise, R.; Rettie, A.; Gonzalez, F.; Tracy, T. Evaluation of atypical cytochrome P450 kinetics with two-substrate models: Evidence that multiple substrates can simultaneously bind to cytochrome P450 active sites. Biochemistry 1998, 37, 4137-4147. [CrossRef] [PubMed]

24. Ellis, L.C.; Hawksworth, G.M.; Weaver, R.J. ATP-dependent transport of statins by human and rat MRP2/Mrp2. Toxicol. Appl. Pharmacol. 2013, 269, 187-194. [CrossRef] [PubMed]

25. Ballatori, N.; Truong, A. Glutathione as a primary osmotic driving force in hepatic bile formation. Am. J. Physiol. Gastroint. Liver Physiol. 1992, 263, G617-G624. [CrossRef] [PubMed]

26. Johnson, D.R.; Habeebu, S.S.; Klaassen, C.D. Increase in bile flow and biliary excretion of glutathione-derived sulfhydryls in rats by drug-metabolizing enzyme inducers is mediated by multidrug resistance protein 2. Toxicol. Sci. 2002, 66, 16-26. [CrossRef] [PubMed]

27. Benz-de Bretagne, I.; Zahr, N.; Le Gouge, A.; Hulot, J.S.; Houillier, C.; Hoang-Xuan, K.; Gyan, E.; Lissandre, S.; Choquet, S.; Le Guellec, C. Urinary coproporphyrin I/(I + III) ratio as a surrogate for MRP2 or other transporter activities involved in methotrexate clearance. Br. J. Clin. Pharmacol. 2014, 78, 329-342. [CrossRef] [PubMed]

28. Benz-de Bretagne, I.; Respaud, R.; Vourc'h, P.; Halimi, J.-M.; Caille, A.; Hulot, J.-S.; Andres, C.R.; Le Guellec, C. Urinary elimination of coproporphyrins is dependent on ABCC2 polymorphisms and represents a potential biomarker of MRP2 activity in humans. J. Biomed. Biotechnol. 2011, 2011, 1-9. [CrossRef] [PubMed]

29. Lai, Y.; Mandlekar, S.; Shen, H.; Holenarsipur, V.K.; Langish, R.; Rajanna, P.; Murugesan, S.; Gaud, N.; Selvam, S.; Date, O. Coproporphyrins in plasma and urine can be appropriate clinical biomarkers to recapitulate drug-drug interactions mediated by organic anion transporting polypeptide inhibition. J. Pharmacol. Exp. Ther. 2016, 358, 397-404. [CrossRef] [PubMed] 
30. Shen, H.; Dai, J.; Liu, T.; Cheng, Y.; Chen, W.; Freeden, C.; Zhang, Y.; Humphreys, W.G.; Marathe, P.; Lai, Y. Coproporphyrins I and III as functional markers of OATP1B activity: In vitro and in vivo evaluation in preclinical species. J. Pharmacol. Exp. Ther. 2016, 357, 382-393. [CrossRef] [PubMed]

31. Bastiaanse, E.L.; Höld, K.M.; Van der Laarse, A. The effect of membrane cholesterol content on ion transport processes in plasma membranes. Cardiovasc. Res. 1992, 33, 272-283. [CrossRef] 